\title{
Against COVID-19: Herbal Theory
}

\author{
Al Fadhil A. Omer* \\ Al Neelain University, Khartoum, Sudan
}

DOI: $\underline{10.36348 / \text { sjbr.2020.v05i05.002 }}$

| Received: 26.04.2020 | Accepted: 03.05.2020 | Published: 09.05.2020

*Corresponding author: Al Fadhil A. Omer

\section{Abstract}

Drugs that slow or kill the novel coronavirus, called severe acute respiratory syndrome coronavirus 2 , could save the lives of severely ill patients, but might also be given prophylactically to protect health care workers and others at high risk of infection. Treatments may also reduce the time patients spend in intensive care units, freeing critical hospital beds. Recovery from the disease depends mainly on the innate immunity of the patient. Patients who suffer much from COVID-19 are children, old people (over 65 years), those patients with chronic diseases (diabetes mellitus, hypertension, liver disease, kidney disease), obese patients, alcoholics, and smokers. About $80 \%$ of corona patients may overcome the infection, and less than 5\% may pass into fatal complications. Patients immunity is the main weapon to fight the disease. The present herbal recipe was selected to support and maintain the innate immunity of the patient against corona virus disease-19. It is composed of 3 herbal materials: bee honey, Nigella sativa (black seed), and Acacia nilotica (Quardh).

Keywords: Covid-19, Bee honey, Nigella sativa, Acacia nilotica, Innate immunity.

Copyright @ 2020: This is an open-access article distributed under the terms of the Creative Commons Attribution license which permits unrestricted use, distribution, and reproduction in any medium for non-commercial use (NonCommercial, or CC-BY-NC) provided the original author and source are credited.

\section{INTRODUCTION}

Scientists and clinicians across the globe have responded to the ongoing coronavirus pandemic with a huge, high-quality global research effort to find a treatment for COVID-19. WHO launches global megatrial of the four most promising coronavirus treatments: a drug combo already used against HIV, a malaria treatment first tested during World War II, a new antiviral whose promise against Ebola fizzled last year. Could any of these drugs hold the key to saving coronavirus disease 2019 (COVID-19) patients from serious harm or death? The World Health Organization (WHO) announced a large global trial, called Solidarity, to find out whether any can treat infections with the new coronavirus for the dangerous respiratory disease. It's an unprecedented effort, an all-out, coordinated push to collect robust scientific data rapidly during a pandemic. The study, which could include many thousands of patients in dozens of countries, has been designed to be as simple as possible so that even hospitals overwhelmed by an onslaught of COVID-19 patients can participate. Drugs that slow or kill the novel coronavirus, called severe acute respiratory syndrome coronavirus 2 , could save the lives of severely ill patients, but might also be given prophylactically to protect health care workers and others at high risk of infection. Treatments may also reduce the time patients spend in intensive care units, freeing critical hospital beds. Scientists have suggested dozens of existing compounds for testing, but WHO is focusing on what it says are the four most promising therapies: an experimental antiviral compound called remdesivir; the malaria medications chloroquine and hydroxychloroquine; a combination of two HIV drugs, lopinavir and ritonavir; and that same combination plus interferon-beta, an immune system messenger that can help cripple viruses. Some data on their use in COVID19 patients have already emerged, the HIV combo failed in a small study in China but WHO believes a large trial is warranted. With about $15 \%$ of COVID-19 patients suffering from severe disease and hospitals being overwhelmed, treatments are desperately needed. So rather than coming up with compounds from scratch that may take years to develop and test, researchers and public health agencies are looking to repurpose drugs already approved for other diseases and known to be largely safe. They're also looking at unapproved drugs that have performed well in animal studies with the other two deadly coronaviruses, which cause severe acute respiratory syndrome and Middle East respiratory syndrome.

\section{HERBAL THEORY}

Until date there is no specific treatment for COVID-19. Recovery from the disease depends mainly on the innate immunity of the patient. The patients who suffer much from COVID-19 are children, old people (over 65 years), those patients with chronic diseases (diabetes mellitus, hypertension, liver disease, kidney 
disease), and obese patients. Almost $80 \%$ of corona patients are able to overcome the infection, and only less than 5\% may die. Patients depend on their own immunity to fight the disease. Hence an herbal recipe was selected to help in supporting the immunity of the patient against corona virus disease-19. The proposed recipe is composed of 3 herbal materials: bee honey, Nigella sativa (black seed), and Acacia nilotica (Quardh).

Chemical composition of bee honey

(1) Carbohydrates: the major portion of honey (82\%). The carbohydrates present are:

a) Monosaccharides: fructose $(38.2 \%)$, and glucose (31\%).

b) Disaccharide (9\%): sucrose, maltose, isomaltose, maltulose, turanose and kojibiose.

c) Oligosaccharides (4.2\%): erlose, theanderose, and panose.

(2) Proteins and amino acids: 18 free amino acids, most abundant is proline.

(3) Enzymes: invertase, amylase, glucose oxidase, catalase, and acid phosphorylase.

(4) Vitamins: Trace amounts of the B vitamins, riboflavin, niacin, folic acid, vitamin B6, pantothenic acid, ascorbic acid (vitamin C), and vitamin $\mathrm{K}$.

(5) Minerals: Calcium, iron, zinc, potassium, phosphorous, magnesium, selenium, chromium, manganese, chlorine, sulpher, and silica.

(6) Antioxidants: flavonoids, ascorbic acid, catalase enzyme, and selenium.

(7) Organic acids (<0.5\%): gluconic acid, acetic acid, butanoic acid, formic acid, citric acid, succinic acid, lactic acid, malic acid, pyroglutamic acid, homogentisic acid, and aromatic acids.

(8) Lipids: glyceride, sterols, and phospholipids.

The chemical substances found in bee honey and may help in treatment of corona patient are: the vitamins, the minerals, and the antioxidants. These substances may maintain the immune system of the patient.

Chemical composition of Nigella sativa (black seed)

(1) The black seeds contain:

a) Volatile and non-volatile oil (containing basic fatty acids, and saponin (melatin).

b) Nigellone antioxidant, arginic acid, and glutathione. The glutathione and nigellone are now prescribed worldwide in the form of capsules to support the immune system.

(2) Thymoquinone relaxes the tracheal muscles and widens the bronchi of the lung, i.e. has an antispasmolytic action.

The substances found in Acacia sativa that may help in treatment of the corona patient are: glutathione, nigellone, melatin, and thymoquinone. Chemical composition of Acacia nilotica (Quardh)
(1) $7 \%$ water, $23 \%$ fat, $7 \%$ fibre, $30 \%$ carbohydrate

(2) Saponins, tannins, flavonoids antioxidant, alkaloids, oxalates and cyanogenic glycosides

(3) Minerals: Calcium, iron, zinc, potassium, phosphorous, magnesium, selenium, chromium, manganese, chlorine, sulpher, sodium, and silica.

(4) Protein amino acids: arginine, leucine, and phenylalanine.

The substances found in Acacia nilotica and help in treatment of the corona patient are: tannins, and flavonoids antioxidants.

Depending on the above facts, Prof. Al Fadhil Al Obeid Omer (Khartoum, Sudan) on 3rd April, 2020, at 10:30 PM announced a recipe proposal pending for a clinical trial among corona virus patients as follows:

*At nasal, oral, and pharyngeal stage

Chew 2-4 seeds of Acacia nilotica for 15 minutes five times daily for one week.

*At tracheal and bronchial stage

Mix $1 / 2 \mathrm{lb}$ pure bee honey with 2 ounces of Nigella sativa powder. On an empty stomach, take One large spoonful from the mixture orally, 4-hourly, and daily for one week.

*Alternatively, take Nigella sativa oil capsules, 40-80 $\mathrm{mg} / \mathrm{kg}$ body wt. daily for one week.

- If the patient is unable to swallow, a stomach tube is needed.

- To combat super aerobic and anaerobic bacterial infections, add ciprofloxacin orally, $\quad 500 \mathrm{mg}$ twice daily for one week.

- Continue on symptomatic treatment.

\section{REFERENCES}

1. Kraemer, M. U., Yang, C. H., Gutierrez, B., Wu, C. H., Klein, B., Pigott, D. M., ... \& Brownstein, J. S. (2020). The effect of human mobility and control measures on the COVID-19 epidemic in China. Science.

2. Zhang, L., Lin, D., Sun, X., Curth, U., Drosten, C., Sauerhering, L., ... \& Hilgenfeld, R. (2020). Crystal structure of SARS-CoV-2 main protease provides a basis for design of improved $\alpha$-ketoamide inhibitors. Science, 368(6489), 409-412.

3. Li, R., Pei, S., Chen, B., Song, Y., Zhang, T., Yang, W., \& Shaman, J. (2020). Substantial undocumented infection facilitates the rapid dissemination of novel coronavirus (SARS-CoV2). Science.

4. Layne, S. P., Hyman, J. M., Morens, D. M., \& Taubenberger, J. K. (2020). New coronavirus outbreak: Framing questions for pandemic prevention.

5. Chinazzi, M., Davis, J. T., Ajelli, M., Gioannini, C., Litvinova, M., Merler, S., \& Viboud, C. (2020). The effect of travel restrictions on the spread of the 2019 novel coronavirus

(COVID-19) outbreak. Science, 368(6489), 395-400. 